\title{
TURBULENT PARAMETERIZATION FOR CCATT-BRAMS BY GPU
}

\author{
Renata S. R. Ruiz ${ }^{1}$, Haroldo F. de Campos Velho ${ }^{1}$, Leandro S. Lessa ${ }^{1}$, Andrea S. Charão ${ }^{2}$ \\ ${ }^{1}$ Instituto Nacional de Pesquisas Espaciais (INPE), Brazil. \\ ${ }^{2}$ Universidade Federal de Santa Maria (UFSM), Brazil
}

renata@1a.inpe.br, Haroldo@lac.inpe.br, Leandro_santos_lessa@yahoo.com.br, andrea@inf.ufsm.br

\begin{abstract}
A strategy to increase the computer code performance is to employ of hybrid computing, combining CPU with GPU or/and FPGA. Two turbulent parameterizations, Smagorinsky and Mellor-Yamada, were codified on GPU for the CCATT-BRAMS code, using the CUDA standard. The results show a good speed-up for the model.
\end{abstract}

\section{RESUMO}

Uma estratégia para aumentar o desempenho de códigos computacionais é o uso de computação híbrida, que combina CPU com GPU e/ou FPGA. Duas parametrizações de turbulência, Smagorinsky e Mellor-Yamada, foram codificadas em GPU para o código CATT-BRAMS:. O padrão CUDA foi usado na implementação. Os resultados mostram um aumento significativo no speed-up do modelo.

\section{INTRODUCTION}

The environmental prediction model CCATT-BRAMS (Freitas et al., 2009; 2013) is a complex computer code, and it is operationally employed by the CPTEC-INPE. This model allows atmospheric simulation with emission, transport, pollutant dispersion, and chemical reactions. The CCATT-BRAMS is already prepared to run on parallel machines. But, depending on the model resolution, this is a very expensive code. This motivates the research to improve its performance. Two parameterizations in the CCATT-BRMAS are codified on GPU: Smagorinsky (1963), and Mellor-Yamada (1982).

\subsection{Smagorinsky's parameterization}

The turbulent fluxes are parameterized using the theory of gradient-flux. The Reynolds tensors are given as following:

$$
\begin{aligned}
& \overline{u_{i}^{\prime} u_{j}^{\prime}}=-\left(K_{m}\right)_{i j}(D)_{j} \\
& (D)_{j}=\partial u_{i} / \partial x_{j}+\partial u_{j} / \partial x_{i} \\
& K_{m}=\left(C s_{z} \Delta z\right)^{2}\left[\left|D_{v}\right|+H(N)\right] f(R)
\end{aligned}
$$

where $c s_{z}$ is a fitting coefficient, $\Delta z$ is the discretization for vertical grid, and $\left|D_{v}\right|$ is the magnitude of the deformation tensor for vertical direction. $H(N)$ and $f\left(R_{i}\right)$ are given by

$$
\begin{aligned}
& H(N)=\sqrt{\max \left(0,-N^{2}\right)} \\
& f\left(R_{\xi}\right)=\max \left\{0 ; 1-\left(K_{h} / K_{m}\right) R_{\xi}\right\}
\end{aligned}
$$


with $N$ being the Brunt-Väisälä frequency, and $\left(K_{h} / K_{m}\right)$ is the ratio between the heat and momentum eddy diffusivities.

\subsection{Mellor-Yamada's parameterization}

This is a 2.5 closure scheme for turbulence, where the third order tensors are parameterized,

$$
\begin{aligned}
& \text { the turbulent } \frac{\partial \overline{\psi_{i}^{\prime} \phi^{\prime}{ }_{j}}}{\partial t}=f\left(\overline{\psi_{i}^{\prime} \phi_{j}^{\prime} \zeta^{\prime}{ }_{k}}, \overline{\psi_{i}^{\prime} \phi_{j}^{\prime}}, \psi_{m}, t\right) \\
& \overline{\psi_{i}^{\prime} \phi^{\prime}{ }_{j} \zeta_{k}^{\prime}} \approx K_{p} \nabla \overline{\psi_{i} \phi^{\prime}{ }_{j}}
\end{aligned}
$$

with a prognostic equation for

$\psi, \phi, \zeta=u, v, w, q, \theta$

where

$$
K_{p}=S_{p} / \sqrt{2 e}, \quad I=\frac{\kappa\left(z+z_{0}\right)}{1+\kappa\left(z+z_{0}\right) I_{\infty}}, \quad I_{\infty}=0.1 \frac{\int_{0}^{d} z \sqrt{2 e} d z}{\int_{0}^{d} \sqrt{2 e} d z}
$$

moisture, and temperature). Key parameters are a length scale $l$, and TKE (e):

where $S_{P}$ are constants suggests by Mellor-Yamada (1982).

\section{CUDA PROGRAMMING ON GPU}

CUDA (Compute Unified Device Architecture) (Nvidia: CUDA, 2012) was developed for the parallel processing for the GPUs produced Nvidia Corporation. The CUDA programming could be understood as an extension of languages like $\mathrm{C}, \mathrm{C}++$, and Fortran, adding qualifiers to functions and data, producing kernels for execution. The job on a kernel is to be divided among thousands of threads, organized into blocks, and grids, with dimensions blockDim and gridDim. The kernel uses indexes blockIdx and threadIdx for defining the job to a thread.

The turbulence routines were codified on Nvidia GPUs: Fermi GTX-580 (512 cores), and Kepler GTX-680 (1536 cores).

\section{RESULTS AND FINAL COMMENTS}

Two horizontal resolutions were evaluated: $40 \mathrm{~km}$ (grid points number: $95 \times 48 \times 38$ ), and 20 $\mathrm{km}(194 \times 100 \times 40)$. Figure 1 shows the speed-up results to Smagorinsky's approach for both resolutions, and for Fermi and Kepler architectures. 


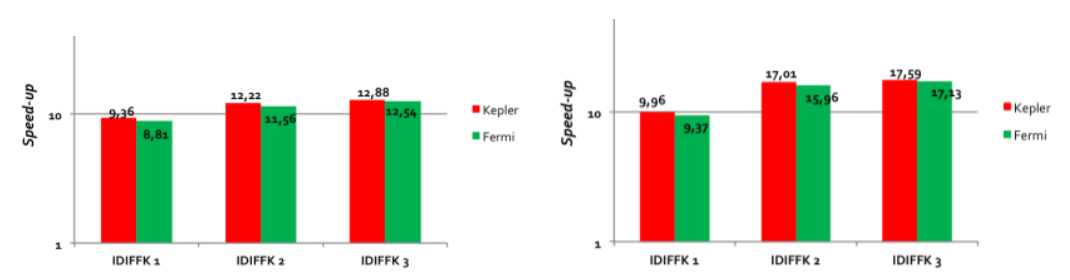

(a)

(b)

Figure 1: GPU execution for Smagorinsky's approach under two resolutions: (a) 40 km, (b) $20 \mathrm{~km}$.

Similarly, the speed-up for the Mellor-Yamada scheme is presented in Figure 2, also considering two resolutions, and Fermi and Kepler architectures performance.

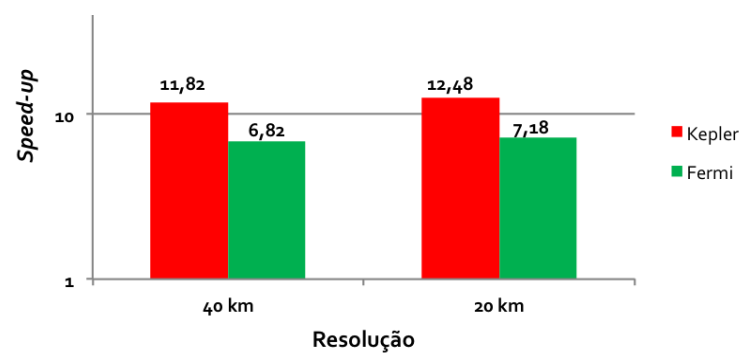

Figure 2: GPU execution for Mellor-Yamada's approach under two resolutions: $40 \mathrm{~km}$, and $20 \mathrm{~km}$.

For the lower resolution worked here, similar speed-up results were obtained for both GPU architectures - the gain for the Mellor-Yamada approach has superior speed-up than the Smagorinsky's parameterization. The results show a tendency to improve the speed-up for a finer resolution.

\section{REFERENCES}

FREITAS, S. R. Et al. The coupled aerosol and tracer transport model to the brazilian developments on the regional atmospheric modeling system (CATT-BRAMS). part 1: Model description and evaluation. Atmos. Chem. Phys. Discuss., v. 9, n. 8, p. 2843-2861, 2009.

LONGO, K. et al. The chemistry CATT-BRAMS model (CCATT-BRAMS 4.5): a regional atmospheric model system for integrated air quality and weather forecasting and research. Geosci. Model Dev., v. 6, 1173-1222, 2013.

SMAGORINSKY, J. General circulation experiments with the primitive equations. I. The Basic Experiment. Mon. Weath. Rev., v. 91, 99-164, 1963.

MELLOR, G. L., YAMADA, T. Development of a turbulence closure model for geophysical fluid problems. Reviews of Geophysics and Space Physics, v.20, n. 4, 851-875, 1982.

NVIDIA Corporation: CUDA Toolkit Documentation, 2012, available at: http://docs.nvidia.com/cuda/. Accessed: July-2013. 